\title{
Transverse-momentum resummation: Higgs boson production at the Tevatron and the LHC
}

\author{
Daniel de Florian, ${ }^{a}$ Giancarlo Ferrera ${ }^{b, c}$ Massimiliano Grazzini ${ }^{d, 1}$ \\ and Damiano Tommasini ${ }^{b, d}$ \\ ${ }^{a}$ Departamento de Fúsica, FCEYN, Universidad de Buenos Aires, \\ 1428 Pabellón 1 Ciudad Universitaria, Capital Federal, Argentina \\ ${ }^{b}$ Dipartimento di Fisica e Astronomia, Università di Firenze and INFN, Sezione di Firenze, \\ I-50019 Sesto Fiorentino, Florence, Italy \\ ${ }^{c}$ Dipartimento di Fisica, Università di Milano and INFN, Sezione di Milano, \\ I-20133 Milan, Italy \\ ${ }^{d}$ Institut für Theoretische Physik, Universität Zürich, \\ CH-8057 Zürich, Switzerland \\ E-mail: deflo@df.uba.ar, giancarlo.ferrera@mi.infn.it, \\ grazzini@physik.uzh.ch, tommasini@fi.infn.it
}

ABStRACT: We consider the transverse-momentum $\left(q_{T}\right)$ distribution of Standard Model Higgs bosons produced by gluon fusion in hadron collisions. At small $q_{T}\left(q_{T} \ll m_{H}, m_{H}\right.$ being the mass of the Higgs boson), we resum the logarithmically-enhanced contributions due to multiple soft-gluon emission to all order in QCD perturbation theory. At intermediate and large values of $q_{T}\left(q_{T} \lesssim m_{H}\right)$, we consistently combine resummation with the known fixed-order results. We use the most advanced perturbative information that is available at present: next-to-next-to-leading logarithmic resummation combined with the next-to-leading fixed-order calculation. We extend previous results including exactly all the perturbative terms up to order $\alpha_{\mathrm{S}}^{4}$ in our computation and, after integration over $q_{T}$, we recover the known next-to-next-to-leading order result for the total cross section. We present numerical results at the Tevatron and the LHC, together with an estimate of the corresponding uncertainties. Our calculation is implemented in an updated version of the numerical code HqT.

KeYwords: Hadronic Colliders, QCD

ARXIV EPRINT: 1109.2109

\footnotetext{
${ }^{1}$ On leave of absence from INFN, Sezione di Firenze, Sesto Fiorentino, Florence, Italy.
} 


\section{Contents}

1 Introduction 1

2 Transverse-momentum resummation $\quad 3$

3 The $q_{T}$ spectrum of the Higgs boson at the Tevatron and the LHC 6

$\begin{array}{lll}4 & \text { Summary } & 15\end{array}$

\section{Introduction}

One of the major tasks of the physics program at high-energy hadron colliders, such as the Fermilab Tevatron and the CERN LHC, is the search for the Higgs boson and the study of its properties.

Gluon-gluon fusion, through a heavy-quark (mainly top-quark) loop, is the main production mechanism of the Standard Model (SM) Higgs boson over the entire range of Higgs boson masses ( $\left.100 \mathrm{GeV} \lesssim m_{H} \lesssim 1 \mathrm{TeV}\right)$ to be investigated at the LHC. At the Tevatron the gluon fusion process, followed by the decay $H \rightarrow W W \rightarrow l^{+} l^{-} \nu \bar{\nu}$, gives the dominant contribution to the Higgs signal in the range of mass $140 \mathrm{GeV} \lesssim m_{H} \lesssim 180 \mathrm{GeV}$. In this mass region, first constraints beyond the LEP lower bound of $114.4 \mathrm{GeV}$ [1] were established: the SM Higgs boson was excluded at $95 \%$ confidence level by CDF and D0 collaborations in the mass range $156 \mathrm{GeV}<m_{H}<177 \mathrm{GeV}$ [2]. The first results of the ATLAS and CMS collaborations presented at EPS 2011 conference [3, 4], and updated for Lepton Photon $2011[5,6]$, dramatically extend the excluded region over most of the mass range between 145 and $466 \mathrm{GeV}$.

The above exclusion relies on accurate theoretical predictions $[7,8]$ for the inclusive $g g \rightarrow H$ cross section, which is now known up to next-to-next-to-leading order (NNLO) [911], with the inclusion of soft-gluon contributions up to next-to-next-to-leading logarithmic accuracy (NNLL) [12], and two-loop electroweak effects [13-17]. ${ }^{1}$

In this paper we consider the transverse momentum $\left(q_{T}\right)$ spectrum of the SM Higgs boson $H$ produced by the gluon fusion mechanism. This observable is of direct importance in the experimental search. A good knowledge of the $q_{T}$ spectrum can help to set up strategies to improve the statistical significance. When studying the $q_{T}$ distribution of the Higgs boson in QCD perturbation theory it is convenient to define two different regions of $q_{T}$. In the large- $q_{T}$ region $\left(q_{T} \sim m_{H}\right)$, where the transverse momentum is of the order of the Higgs boson mass $m_{H}$, perturbative QCD calculations based on the truncation of the perturbative series at a fixed order in $\alpha_{\mathrm{S}}$ are theoretically justified. In this region, the

\footnotetext{
${ }^{1}$ Updated predictions for the inclusive Higgs production cross sections at the LHC are presented in ref. [18].
} 
QCD radiative corrections are known up to the next-to-leading order (NLO) [19-21] and QCD corrections beyond the NLO are evaluated in ref. [22], by implementing threshold resummation at the next-to-leading logarithmic (NLL) level.

In the small- $q_{T}$ region $\left(q_{T} \ll m_{H}\right)$, where the bulk of the events is produced, the convergence of the fixed-order expansion is spoiled by the presence of large logarithmic terms, $\alpha_{\mathrm{S}}^{n} \ln ^{m}\left(m_{H}^{2} / q_{T}^{2}\right)$. To obtain reliable predictions, these logarithmically-enhanced terms have to be systematically resummed to all perturbative orders [23-36]. It is then important to consistently match the resummed and fixed-order calculations at intermediate values of $q_{T}$, in order to obtain accurate QCD predictions for the entire range of transverse momenta.

The resummation of the logarithmically enhanced terms is effectively (approximately) performed by standard Monte Carlo event generators. In particular, MC@NLO [37] and POWEG $[38,39]$ combine soft-gluon resummation through the parton shower with the leading order (LO) result valid at large $q_{T}$, thus achieving a result with formal NLO accuracy.

The numerical program HqT [35] implements soft-gluon resummation up to NNLL accuracy [40] combined with fixed-order perturbation theory up to NLO in the large- $q_{T}$ region [21]. The program is used by the Tevatron and LHC experimental collaborations to reweight the $q_{T}$ spectrum of the Monte Carlo event generators used in the analysis and is thus of direct relevance in the Higgs boson search.

The program HqT is based on the transverse-momentum resummation formalism described in refs. [34-36], which is valid for a generic process in which a high-mass system of non strongly-interacting particles is produced in hadron-hadron collisions. The method has so far been applied to the production of the SM Higgs boson [35, 41, 42], single vector bosons [43, 44], $W W$ [45] and $Z Z$ [46] pairs, slepton pairs [47], and Drell-Yan lepton pairs in polarized collisions [48-51].

In this paper we update and extend the phenomenological analysis presented in ref. [35]. In particular, we implement the exact value of the NNLO hard-collinear coefficients $\mathcal{H}_{N}^{H(2)}$ computed in ref. $[52,53]$, and the recently derived value of the NNLL coefficient $A^{(3)}$ [54].

We use the most advanced perturbative information that is available at present: NNLL resummation at small $q_{T}$ and the fixed-order NLO calculation at large $q_{T}$. We present numerical results for Higgs production at the Tevatron Run II and at the LHC and we perform a detailed study of the perturbative uncertainties. We also consider the normalized $q_{T}$ spectrum and discuss its theoretical uncertainties. Our calculation for the $q_{T}$ spectrum is implemented in the updated version of the numerical code HqT, which can be downloaded from [55]. Other phenomenological studies of the Higgs boson $q_{T}$ distribution, which combine resummed and fixed-order perturbative results at various levels of theoretical accuracy, can be found in refs. [56]-[63].

The paper is organized as follows. In section 2 we briefly review the resummation formalism of refs. [34-36] and its application to Higgs boson production. In section 3 we present numerical results for Higgs boson production at the Tevatron and the LHC. In section 4 we summarize our results. 


\section{Transverse-momentum resummation}

In this section we briefly recall the main points of the transverse-momentum resummation approach proposed in refs. [34-36]. We consider the specific case of a Higgs boson $H$ produced by gluon fusion. As recently pointed out in ref. [36], the gluon fusion $q_{T}$-resummation formula has a different structure than the resummation formula for $q \bar{q}$ annihilation. The difference originates from the collinear correlations that are a specific feature of the perturbative evolution of colliding hadron into gluon partonic initial states. These gluon collinear correlations produce, in the small- $q_{T}$ region, coherent spin correlations between the helicity states of the initial-state gluons and definite azimuthal-angle correlations between the final-states particles of the observed high-mass system. Both these kinds of correlations have no analogue for $q \bar{q}$ annihilation processes in the small- $q_{T}$ region. In the case of Higgs boson production, being $H$ a spin-0 scalar particle, the azimuthal correlations vanishes and only gluon spin correlations are present [36].

We consider the inclusive hard-scattering process

$$
h_{1}\left(p_{1}\right)+h_{2}\left(p_{2}\right) \rightarrow H\left(m_{H}, q_{T}\right)+X,
$$

where $h_{1}$ and $h_{2}$ are the colliding hadrons with momenta $p_{1}$ and $p_{2}, m_{H}$ and $q_{T}$ are the Higgs boson mass and transverse momentum respectively, and $X$ is an arbitrary and undetected final state.

According to the QCD factorization theorem the corresponding transverse-momentum differential cross section $d \sigma_{H} / d q_{T}^{2}$ can be written as

$$
\begin{aligned}
\frac{d \sigma_{H}}{d q_{T}^{2}}\left(q_{T}, m_{H}, s\right)=\sum_{a, b} \int_{0}^{1} d x_{1} \int_{0}^{1} d & x_{2} f_{a / h_{1}}\left(x_{1}, \mu_{F}^{2}\right) f_{b / h_{2}}\left(x_{2}, \mu_{F}^{2}\right) \times \\
\times & \frac{d \hat{\sigma}_{H, a b}}{d q_{T}^{2}}\left(q_{T}, m_{H}, \hat{s} ; \alpha_{\mathrm{S}}\left(\mu_{R}^{2}\right), \mu_{R}^{2}, \mu_{F}^{2}\right),
\end{aligned}
$$

where $f_{a / h}\left(x, \mu_{F}^{2}\right)(a=q, \bar{q}, g)$ are the parton densities of the colliding hadron $h$ at the factorization scale $\mu_{F}, d \hat{\sigma}_{H, a b} / d q_{T}^{2}$ are the perturbative QCD partonic cross sections, $s$ $\left(\hat{s}=x_{1} x_{2} s\right)$ is the square of the hadronic (partonic) centre-of-mass energy, and $\mu_{R}$ is the renormalization scale. ${ }^{2}$

In the region where $q_{T} \sim m_{H}$, the QCD perturbative series is controlled by a small expansion parameter, $\alpha_{\mathrm{S}}\left(m_{H}\right)$, and fixed-order calculations are theoretically justified. In this region, the QCD radiative corrections are known up to NLO [19-21]. In the small- $q_{T}$ region $\left(q_{T} \ll m_{H}\right)$, the convergence of the fixed-order perturbative expansion is ruined by the presence of powers of large logarithmic terms, $\alpha_{\mathrm{S}}^{n} \ln ^{m}\left(m_{H}^{2} / q_{T}^{2}\right)($ with $1 \leq m \leq 2 n-1)$. To obtain reliable predictions these terms have to be resummed to all orders.

We perform the resummation at the level of the partonic cross section, which is decomposed as

$$
\frac{d \hat{\sigma}_{H, a b}}{d q_{T}^{2}}=\frac{d \hat{\sigma}_{H, a b}^{\text {(res. })}}{d q_{T}^{2}}+\frac{d \hat{\sigma}_{H, a b}^{(\text {fin. })}}{d q_{T}^{2}} .
$$

\footnotetext{
${ }^{2}$ Throughout the paper we use parton densities $f\left(x, \mu_{F}^{2}\right)$ and running coupling $\alpha_{\mathrm{S}}\left(\mu_{R}^{2}\right)$ as defined in the $\overline{\mathrm{MS}}$ scheme.
} 
The first term on the right-hand side includes all the logarithmically-enhanced contributions, at small $q_{T}$, and has to be evaluated to all orders in $\alpha_{\mathrm{S}}$. The second term is free of such contributions and can thus be computed at fixed order in perturbation theory. To correctly take into account the kinematic constraint of transverse-momentum conservation, the resummation program has to be carried out in the impact parameter space $b$. Using the Bessel transformation between the conjugate variables $q_{T}$ and $b$, the resummed component $d \hat{\sigma}_{H, a c}^{(\text {res. })}$ can be expressed as

$$
\frac{d \hat{\sigma}_{H, a c}^{\text {(res. })}}{d q_{T}^{2}}\left(q_{T}, m_{H}, \hat{s} ; \alpha_{\mathrm{S}}\left(\mu_{R}^{2}\right), \mu_{R}^{2}, \mu_{F}^{2}\right)=\int_{0}^{\infty} d b \frac{b}{2} J_{0}\left(b q_{T}\right) \mathcal{W}_{a c}^{H}\left(b, m_{H}, \hat{s} ; \alpha_{\mathrm{S}}\left(\mu_{R}^{2}\right), \mu_{R}^{2}, \mu_{F}^{2}\right),
$$

where $J_{0}(x)$ is the 0th-order Bessel function. The resummation structure of $\mathcal{W}_{a c}^{H}$ can be organized in exponential form considering the Mellin $N$-moments $\mathcal{W}_{N}^{H}$ of $\mathcal{W}^{H}$ with respect to the variable $z=m_{H}^{2} / \hat{s}$ at fixed $m_{H},^{3}$

$$
\begin{aligned}
\mathcal{W}_{N}^{H}\left(b, m_{H} ; \alpha_{\mathrm{S}}\left(\mu_{R}^{2}\right), \mu_{R}^{2}, \mu_{F}^{2}\right)=\mathcal{H}_{N}^{H}\left(m_{H}, \alpha_{\mathrm{S}}\left(\mu_{R}^{2}\right) ; m_{H}^{2} / \mu_{R}^{2}, m_{H}^{2} / \mu_{F}^{2}, m_{H}^{2} / Q^{2}\right) \\
\times \exp \left\{\mathcal{G}_{N}\left(\alpha_{\mathrm{S}}\left(\mu_{R}^{2}\right), L ; m_{H}^{2} / \mu_{R}^{2}, m_{H}^{2} / Q^{2}\right)\right\},
\end{aligned}
$$

were we have defined the logarithmic expansion parameter $L \equiv \ln \left(Q^{2} b^{2} / b_{0}^{2}\right)$, and $b_{0}=2 e^{-\gamma_{E}}$ $\left(\gamma_{E}=0.5772 \ldots\right.$ is the Euler number $)$.

The scale $Q \sim m_{H}$, appearing in the right-hand side of eq. (2.5), named resummation scale [35], parameterizes the arbitrariness in the resummation procedure. As a matter of fact the argument of the resummed logarithms can always be rescaled as $\ln \left(m_{H}^{2} b^{2}\right)=$ $\ln \left(Q^{2} b^{2}\right)+\ln \left(m_{H}^{2} / Q^{2}\right)$ (as long as $Q \sim m_{H}$ and independent of $b$ ). Although $\mathcal{W}_{N}^{H}$ (i.e., the product $\left.\mathcal{H}_{N}^{H} \times \exp \left\{\mathcal{G}_{N}\right\}\right)$ does not depend on $Q$ when evaluated to all perturbative orders, its explicit dependence on $Q$ appears when $\mathcal{W}_{N}^{H}$ is computed by truncation of the resummed expression at some level of logarithmic accuracy (see eq. (2.6) below). As in the case of $\mu_{R}$ and $\mu_{F}$, variations of $Q$ around $m_{H}$ can thus be used to estimate the uncertainty from yet uncalculated logarithmic corrections at higher orders.

The form factor $\exp \left\{\mathcal{G}_{N}\right\}$ is universal (process independent) ${ }^{4}$ and contains all the terms $\alpha_{\mathrm{S}}^{n} L^{m}$ with $1 \leq m \leq 2 n$, that order-by-order in $\alpha_{\mathrm{S}}$ are logarithmically divergent as $b \rightarrow \infty$ (or, equivalently, $q_{T} \rightarrow 0$ ). Furthermore, due to the exponentiation property, all the logarithmic contributions to $\mathcal{G}_{N}$ with $n+2 \leq m \leq 2 n$ are vanishing. The exponent $\mathcal{G}_{N}$ can be systematically expanded as

$$
\begin{aligned}
\mathcal{G}_{N}\left(\alpha_{\mathrm{S}}, L ; m_{H}^{2} / \mu_{R}^{2}, m_{H}^{2} / Q^{2}\right)= & L g^{(1)}\left(\alpha_{\mathrm{S}} L\right)+g_{N}^{(2)}\left(\alpha_{\mathrm{S}} L ; m_{H}^{2} / \mu_{R}^{2}, m_{H}^{2} / Q^{2}\right) \\
& +\frac{\alpha_{\mathrm{S}}}{\pi} g_{N}^{(3)}\left(\alpha_{\mathrm{S}} L ; m_{H}^{2} / \mu_{R}^{2}, m_{H}^{2} / Q^{2}\right)+\mathcal{O}\left(\alpha_{\mathrm{S}}^{n} L^{n-2}\right)
\end{aligned}
$$

\footnotetext{
${ }^{3}$ For the sake of simplicity we write the resummation formulae only for the specific case of the diagonal terms in the flavour space. In general, the exponential is replaced by an exponential matrix with respect to the partonic indeces (a detailed discussion of the general case can be found in ref. [35]).

${ }^{4}$ It only depends on the partonic channel that produces the Born cross section. It is thus usually called quark or gluon Sudakov form factor.
} 
where the term $L g^{(1)}$ resums the leading logarithmic (LL) contributions $\alpha_{\mathrm{S}}^{n} L^{n+1}$, the function $g_{N}^{(2)}$ includes the NLL contributions $\alpha_{\mathrm{S}}^{n} L^{n}$ [33], $g_{N}^{(3)}$ controls the NNLL terms $\alpha_{\mathrm{S}}^{n} L^{n-1}[40,54]$ and so forth. The explicit form of the functions $g^{(1)}, g_{N}^{(2)}$ and $g_{N}^{(3)}$ can be found in ref. [35].

The process dependent function $\mathcal{H}_{N}^{H}$ does not depend on the impact parameter $b$ and it includes all the perturbative terms that behave as constants as $b \rightarrow \infty$. It can thus be expanded in powers of $\alpha_{\mathrm{S}}=\alpha_{\mathrm{S}}\left(\mu_{R}^{2}\right)$ :

$$
\begin{aligned}
& \mathcal{H}_{N}^{H}\left(m_{H}, \alpha_{\mathrm{S}} ; m_{H}^{2} / \mu_{R}^{2}, m_{H}^{2} / \mu_{F}^{2}, m_{H}^{2} / Q^{2}\right) \\
&=\sigma_{H}^{(0)}\left(\alpha_{\mathrm{S}}, m_{H}\right)[1+\frac{\alpha_{\mathrm{S}}}{\pi} \mathcal{H}_{N}^{H,(1)}\left(m_{H}^{2} / \mu_{F}^{2}, m_{H}^{2} / Q^{2}\right) \\
&\left.+\left(\frac{\alpha_{\mathrm{S}}}{\pi}\right)^{2} \mathcal{H}_{N}^{H,(2)}\left(m_{H}^{2} / \mu_{R}^{2}, m_{H}^{2} / \mu_{F}^{2}, m_{H}^{2} / Q^{2}\right)+\mathcal{O}\left(\alpha_{\mathrm{S}}^{3}\right)\right],
\end{aligned}
$$

where $\sigma_{H}^{(0)}\left(\alpha_{\mathrm{S}}, m_{H}\right)$ is the partonic cross section at the Born level. The first order $\mathcal{H}_{N}^{H,(1)}[64$, 65] and the second order $\mathcal{H}_{N}^{H,(2)}$ [52,53] coefficients in eq. (2.7), for the case of Higgs boson production in the large- $M_{t}$ approximation, are known.

To reduce the impact of unjustified higher-order contributions in the large- $q_{T}$ region, the logarithmic variable $L$ in eq. (2.5), which diverges for $b \rightarrow 0$, is actually replaced by $\widetilde{L} \equiv \ln \left(Q^{2} b^{2} / b_{0}^{2}+1\right)[35,41]$. The variables $L$ and $\widetilde{L}$ are equivalent when $Q b \gg 1$ (i.e. at small values $q_{T}$ ), but they lead to a different behaviour of the form factor at small values of $b$. An important consequence of this replacement is that, after inclusion of the finite component (see eq. (2.8)), we exactly recover the fixed-order perturbative value of the total cross section upon integration of the $q_{T}$ distribution over $q_{T}$ (i.e., the contribution of the resummed terms vanishes upon integration over $q_{T}$ ). We note that there are infinite ways of modifying the logarithmic term in order to achieve this goal. A similar effect could have been obtained for example by the replacement $L \rightarrow \tilde{L}=1 / p \times \ln \left[\left(Q^{2} b^{2} / b_{0}^{2}\right)^{p}+1\right]$. Considering the small- $b$ region, the quantitative effect of changing the resummation scale $Q$ is similar to a change of the parameter $p$; by performing $Q$ variations we can thus also have an idea of the arbitrariness of the resummation procedure.

The finite component of the transverse-momentum cross section $d \sigma_{H}^{(\text {fin.) }}$ (see eq. (2.3)) does not contain large logarithmic terms in the small- $q_{T}$ region, it can thus be evaluated by truncation of the perturbative series at a given fixed order. In practice it is computed as follows

$$
\left[\frac{d \hat{\sigma}_{H, a b}^{(\text {fin. })}}{d q_{T}^{2}}\right]_{\text {f.o. }}=\left[\frac{d \hat{\sigma}_{H, a b}}{d q_{T}^{2}}\right]_{\text {f.o. }}-\left[\frac{d \hat{\sigma}_{H, a b}^{\text {(res. })}}{d q_{T}^{2}}\right]_{\text {f.o. }},
$$

where we have introduced the subscript f.o. to denote the perturbative truncation of the various terms. This matching procedure combines the resummed and the finite component of the partonic cross section without double-counting in the intermediate $q_{T}$-region and allows us to achieve a prediction with uniform theoretical accuracy over the entire range of transverse momenta.

In summary, to carry out the resummation at NLL+LO accuracy, we need the inclusion of the functions $g^{(1)}, g_{N}^{(2)}, \mathcal{H}_{N}^{H,(1)}$, in eqs. (2.6), (2.7), together with the evaluation of the 
finite component at LO (i.e. at $\left.\mathcal{O}\left(\alpha_{\mathrm{S}}\right)\right)$ in eq. (2.8); the addition of the functions $g_{N}^{(3)}$ and $\mathcal{H}_{N}^{H,(2)}$, together with the finite component at NLO (i.e. at $\mathcal{O}\left(\alpha_{\mathrm{S}}^{2}\right)$ ) leads to the NNLL+NLO accuracy. ${ }^{5}$ We point out that our best theoretical prediction (NNLL+NLO) includes the full NNLO perturbative contribution in the small- $q_{T}$ region plus the NLO correction at large- $q_{T}$. In particular, the NNLO result for the total cross section is exactly recovered upon integration over $q_{T}$ of the differential cross section $d \sigma_{H} / d q_{T}$ at NNLL+NLO accuracy.

Finally we recall that the resummed form factor $\exp \left\{\mathcal{G}_{N}\left(\alpha_{\mathrm{S}}\left(\mu_{R}^{2}\right), \widetilde{L}\right)\right\}$ has a singular behaviour, related to the presence of the Landau pole in the QCD running coupling, at the values of $b$ where $\alpha_{\mathrm{S}}\left(\mu_{R}^{2}\right) \widetilde{L} \geq \pi / \beta_{0}$ ( $\beta_{0}$ is the first-order coefficient of the QCD $\beta$ function). To perform the inverse Bessel transformation with respect to the impact parameter $b$ a prescription is thus necessary. We deal with this singularity by using the regularization prescription of refs. [70, 71]: the singularity is avoided by deforming the integration contour in the complex $b$ space.

\section{The $q_{T}$ spectrum of the Higgs boson at the Tevatron and the LHC}

In this section we consider Higgs boson production by gluon fusion at the Tevatron $(\sqrt{s}=$ $1.96 \mathrm{TeV})$ and the $\mathrm{LHC}(\sqrt{s}=7 \mathrm{TeV}$ and $14 \mathrm{TeV})$. We present our resummed results at NNLL+NLO accuracy, and we compare them with the NLL+LO results. For the Tevatron we choose $m_{H}=165 \mathrm{GeV}$. For the LHC at $\sqrt{s}=7$ and $\sqrt{s}=14 \mathrm{TeV}$ we fix $m_{H}=165 \mathrm{GeV}$ and $m_{H}=125 \mathrm{GeV}$, respectively.

The results we present in this section are obtained with an updated version of the numerical code HqT [55]. The new version of this code was improved with respect to the one used in ref. [35]. The main differences regard the implementation of the second-order coefficients $\mathcal{H}_{N}^{H,(2)}$ computed in ref. [52] (the numerical results in ref. [35] were obtained by using a reasonable approximation of this coefficient) and the use of the recently derived value of the coefficient $A^{(3)}[54]$ which contributes to the NNLL function $g_{N}^{(3)}$ (the results in ref. [35] were obtained by using the $A^{(3)}$ value from threshold resummation [72]). We have checked the quantitative effect of the exact values of $\mathcal{H}^{H,(2)}$ and $A^{(3)}$ at the Tevatron and the LHC. We find that the effect is generally small (at the level of about $1-2 \%$ at the $\mathrm{LHC}$ at $14 \mathrm{TeV}, 2-3 \%$ at the Tevatron, and at the $\mathrm{LHC}$ with $7 \mathrm{TeV}$ ). We also find that the exact values of $\mathcal{H}^{H,(2)}$ and $A^{(3)}$ have the same qualitative impact: they make the $q_{T}$-spectrum (slightly) harder.

The calculation is performed strictly in the large- $M_{t}$ approximation. This is known to be a good approximation for the $q_{T}$ spectrum, provided that $q_{T}$ is not too large $\left(q_{T} \lesssim M_{t}\right)$ [73]. For very large transverse momenta the large- $M_{t}$ approximation is bound to fail, since the QCD radiation accompanying the Higgs boson becomes sensitive to the heavy-quark loop. Considering the normalized $q_{T}$ spectrum, for $q_{T} \lesssim M_{t}$, corrections beyond the large- $M_{t}$ approximation are expected to be at the few percent level.

The hadronic $q_{T}$ cross section at NNLL+NLO (NLL+LO) accuracy is computed by using NNLO (NLO) parton distributions functions (PDFs) with $\alpha_{\mathrm{S}}\left(\mu_{R}^{2}\right)$ evaluated at 3-

\footnotetext{
${ }^{5}$ The evaluation of the second-order coefficient $\mathcal{H}_{N}^{H,(2)}$ for complex values of $N$, necessary to perform the inverse Mellin transform, is obtained using the numerical results of ref. [66-69].
} 
loop (2-loop) order. This choice of the order of the parton densities and $\alpha_{\mathrm{S}}$ is fully justified both in the small- $q_{T}$ region (where the calculation of the partonic cross section includes the complete NNLO (NLO) result and is controlled by NNLL (NLL) resummation) and in the intermediate- $q_{T}$ region (where the calculation is constrained by the value of the NNLO (NLO) total cross section). Recent sets of parton densities, which are obtained by analyses of various collaborations, are presented in refs. [74-80]. Since the main purpose of our work is the study of the $q_{T}$ distribution up to the NNLL+NLO, we consider here only the PDFs sets of refs. [77-80], which provide NNLO parton densities with $N_{f}=5$ (effectively) massless quarks. Moreover, to avoid multiple presentations of similar results, we use the MSTW2008 parton densities unless otherwise stated (the results in ref. [35] were obtained by using the MRST2004 set [81]).

As discussed in section 2, the resummed calculation depends on the factorization and renormalization scales and on the resummation scale $Q$. Our convention to compute factorization and renormalization scale uncertainties is to consider independent variations of $\mu_{F}$ and $\mu_{R}$ by a factor of two around the central values $\mu_{F}=\mu_{R}=m_{H}$ (i.e. we consider the range $m_{H} / 2 \leq\left\{\mu_{F}, \mu_{R}\right\} \leq 2 m_{H}$ ), with the constraint $0.5 \leq \mu_{F} / \mu_{R} \leq 2$. Similarly, we follow ref. [35] and choose $Q=m_{H} / 2$ as central value of the resummation scale, considering scale variations in the range $m_{H} / 4<Q<m_{H}$.

In figure 1 (left panels) we present the NLL+LO $q_{T}$ spectrum of a Higgs boson at the Tevatron, and at the LHC with $\sqrt{s}=7 \mathrm{TeV}$ and $\sqrt{s}=14 \mathrm{TeV}$. The NLL+LO result (solid lines) at the default scales $\left(\mu_{F}=\mu_{R}=m_{H}, Q=m_{H} / 2\right)$ are compared with the corresponding LO results (dashed lines). The LO finite component of the spectrum (see eq. (2.3)) is also shown for comparison (dotted lines). We see that the LO result diverges to $+\infty$ as $q_{T} \rightarrow 0$. The resummation of the small- $q_{T}$ logarithms leads to a well-behaved distribution: it vanishes as $q_{T} \rightarrow 0$, has a kinematical peak, and tends to the corresponding LO result at large values of $q_{T}$. The finite component smoothly vanishes as $q_{T} \rightarrow 0$ but gives a sizable contribution to the NLL+LO result in the low- $q_{T}$ region.

The results in the right panels of figure 1 are analogous to those in the left panels although systematically at one order higher. The $q_{T}$ spectrum at NNLL+NLO accuracy (solid line) is compared with the NLO result (dashed line) and with the NLO finite component of the spectrum (dotted line). The NLO result diverges to $-\infty$ as $q_{T} \rightarrow 0$ and, at small values of $q_{T}$, it has an unphysical peak (the top of the peak is above the vertical scale of the plot) that is produced by the numerical compensation of negative leading and positive subleading logarithmic contributions. In the region of intermediate values of $q_{T}$ (say, around $50 \mathrm{GeV}$ ), the difference between the NNLL+NLO and NLO results gives a sizable contribution with respect to the NLO finite component. This difference is produced by the logarithmic terms (at NNLO and beyond NNLO) that are included in the resummed calculation at NNLL accuracy. At large values of $q_{T}$ the contribution of the NLO finite component noticeably increases. This behaviour indicates that the logarithmic terms are no longer dominant and that the resummed calculation cannot improve upon the predictivity of the fixed-order expansion.

Comparing the left and right panels of figure 1, we see that the size of the $q_{T}$ spectrum increases at NNLL+NLO accuracy with respect to the NLL+LO accuracy. The height of 


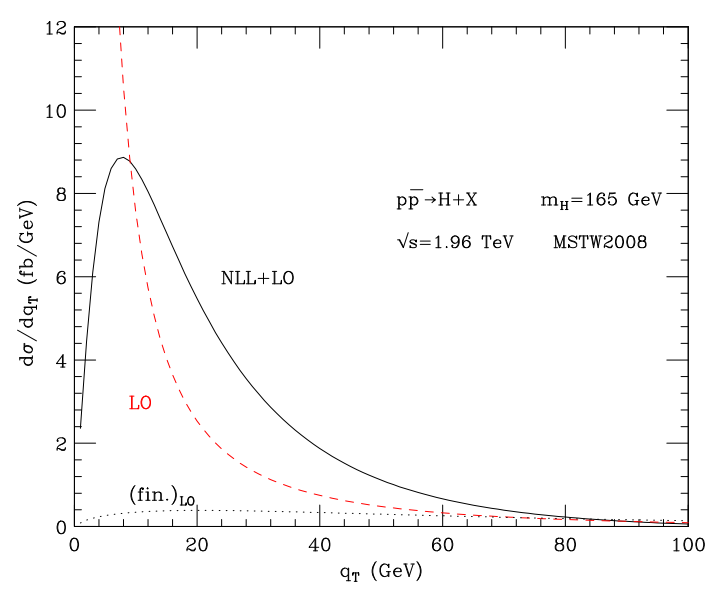

(a)

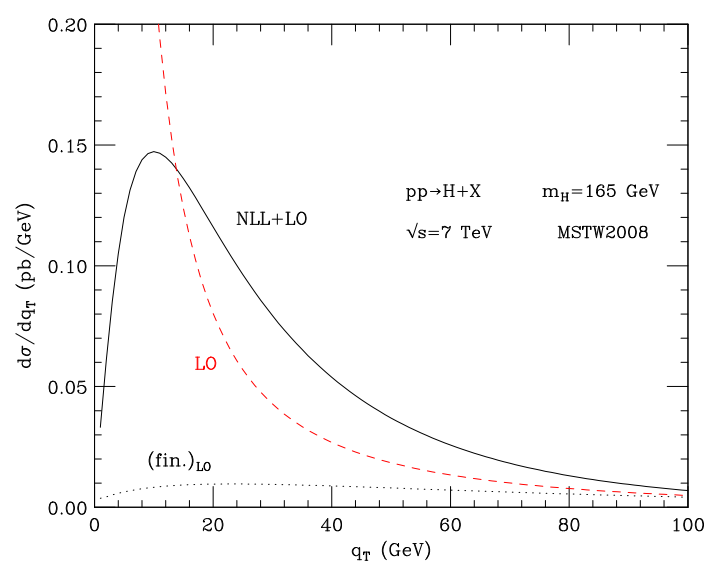

(c)

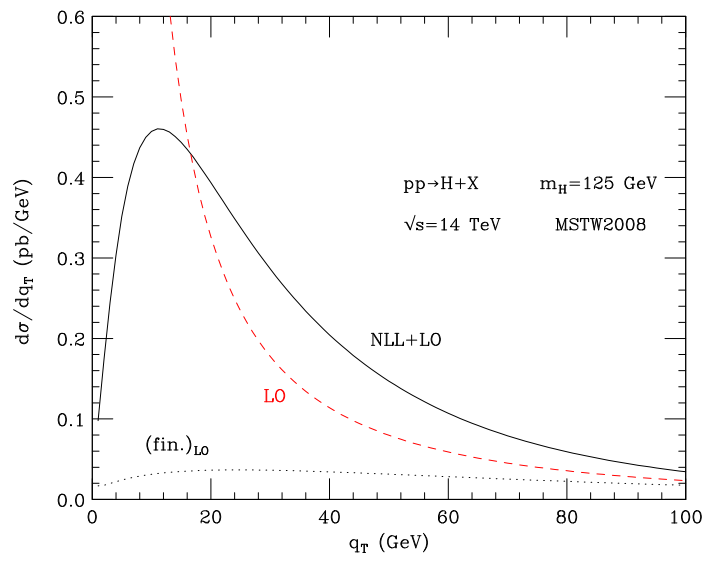

(e)

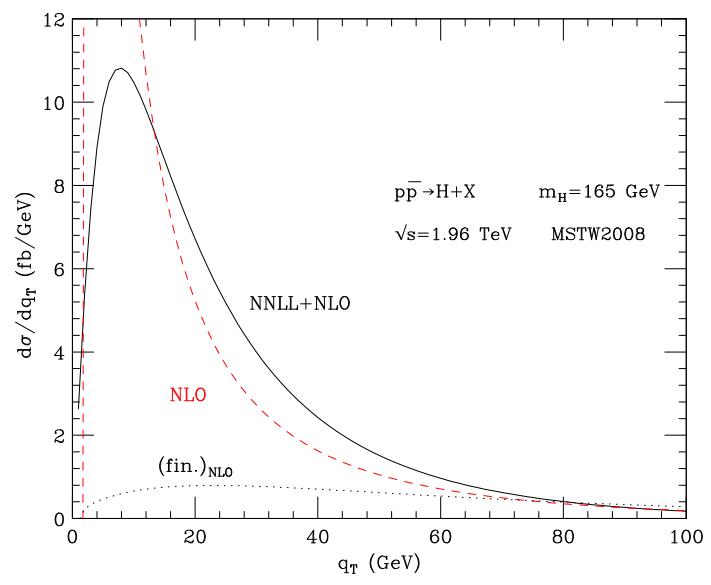

(b)

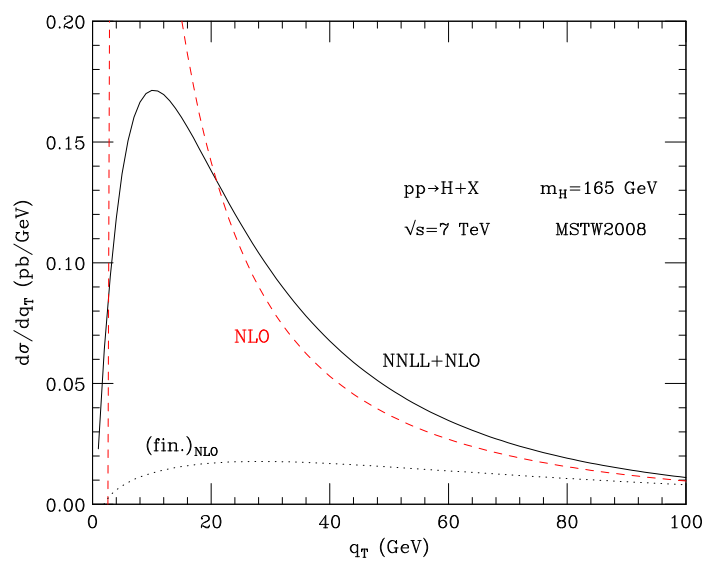

(d)

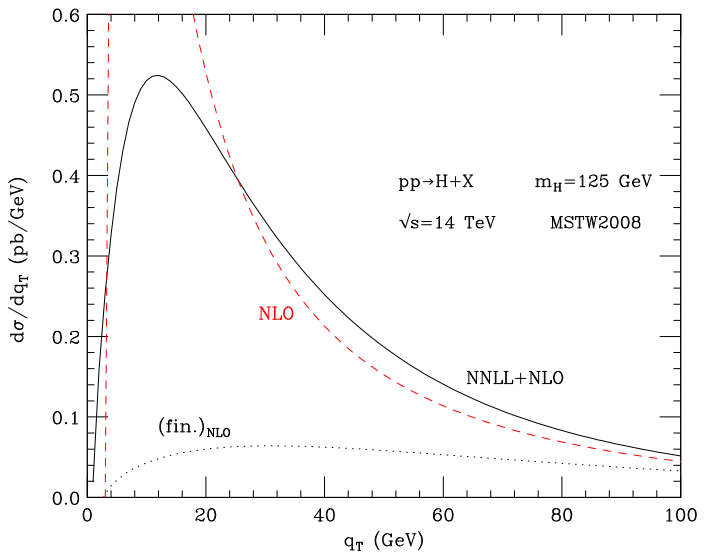

(f)

Figure 1. The $q_{T}$ spectrum of Higgs bosons at the Tevatron and the LHC. Results shown are at NLL+LO (left panels) and NNLL+NLO (right panels) accuracy. Each result is compared to the corresponding fixed-order result (dashed line) and to the finite component (dotted line) in eq. (2.8). 
the peak at NNLL+NLO is larger than at NLL+LO. The NNLO total cross section, which fixes the value of the $q_{T}$ integral of our NNLL+NLO result, is larger than the NLO total cross section (by about $30 \%$ at the Tevatron and $25 \%$ at the LHC). This is due to the positive contribution of both the NNLO terms at small $q_{T}$ (the $\mathcal{H}_{N}^{H,(2)}$ coefficient of the the $\mathcal{H}_{N}^{H}$ function and the $g_{N}^{(3)}$ function in the Sudakov form factor) and the NLO finite component at intermediate and large values of $q_{T}$.

Comparing figure 1(a), 1(b) with figure 1(c), 1(d) and Fig 1(e), 1(f) we see that the spectrum is harder at the LHC than at the Tevatron. The peak of the NNLL+NLO curve moves from $q_{T} \sim 8 \mathrm{GeV}$ at the Tevatron, to $q_{T} \sim 10 \mathrm{GeV}$ at the LHC at $\sqrt{s}=7 \mathrm{TeV}$, to $q_{T} \sim 12 \mathrm{GeV}$ at the LHC at $\sqrt{s}=14 \mathrm{TeV}$.

In figure 2 we show the scale dependence of the NLL+LO (dashed lines) and NNLL+NLO (solid lines) results. In the left panels we consider variations of the renormalization and factorization scales. The bands are obtained by varying $\mu_{R}$ and $\mu_{F}$ as previously described in this section. We note that, in the region of small and intermediate transverse momenta $\left(q_{T} \lesssim 70 \mathrm{GeV}\right)$, the NNLL+NLO and NLL+LO bands overlap. This feature, which is not present in the case of the fixed-order perturbative results at LO and NLO, confirms the importance of resummation to achieve a stable perturbative prediction. In the region of small and intermediate values of $q_{T}$, we observe a sensible reduction of the scale dependence going from NLL+LO to NNLL+NLO accuracy. At the peak the reduction is from $\pm 20 \%$ to $\pm 13 \%$ at the Tevatron, and from $\pm 11 \%$ to $\pm 8 \%( \pm 12 \%$ to $\pm 7 \%)$ at the LHC with $\sqrt{s}=7(\sqrt{s}=14) \mathrm{TeV}$. Although $\mu_{R}$ and $\mu_{F}$ are varied independently, we find that the dependence on $\mu_{R}$ dominates at any value of $q_{T}$.

We point out that the $q_{T}$ region where resummed perturbative predictions are definitely significant is a wide region from intermediate to relatively-small (say, close to the peak of the distribution) values of $q_{T}$. In fact, at very small values of $q_{T}$ (e.g. $q_{T} \lesssim 10 \mathrm{GeV}$ ) the size of non-perturbative effects is expected to be important, ${ }^{6}$ while in the high- $q_{T}$ region (e.g. $q_{T} \gtrsim m_{H} \mathrm{GeV}$ ) the resummation of the logarithmic terms cannot improve the predictivity of the fixed-order perturbative expansion. The inset plots in the figure show the region from intermediate to large values of $q_{T}$. At large $q_{T}$, the NLL+LO and NNLL+NLO results deviate from each other, and the deviation increases as $q_{T}$ increases. As previously stated, this behaviour is not particularly worrying since, in the large- $q_{T}$ region, the resummed results loose their predictivity and should be replaced by customary fixed-order results.

In the right panels of figure 2 we consider resummation scale variations. The bands are obtained by fixing $\mu_{R}=\mu_{F}=m_{H}$ and varying $Q$ between $m_{H} / 4$ and $m_{H}$. Performing variations of the resummation scale, we can get further insight on the size of yet uncalculated higher-order logarithmic contributions at small and intermediate values of $q_{T}$. We find that, in the region of the peak, at the Tevatron the scale dependence at NNLL+NLO $(\mathrm{NLL}+\mathrm{LO})$ is about $\pm 4 \%( \pm 10 \%)$. At the LHC with $\sqrt{s}=7 \mathrm{TeV}$ the scale dependence at $\mathrm{NNLL}+\mathrm{NLO}(\mathrm{NLL}+\mathrm{LO})$ is about $\pm 3 \%( \pm 8 \%)$ and at $\sqrt{s}=14$ it is about $\pm 3 \%( \pm 13 \%)$.

Comparing the left and right panels of figure 2, we see that, in the small and intermediate $q_{T}$ region, at NNLL+NLO accuracy, the factorization and renormalization scale dependence is definitely larger than the resummation scale dependence.

\footnotetext{
${ }^{6}$ See the discussion at the end of this section.
} 


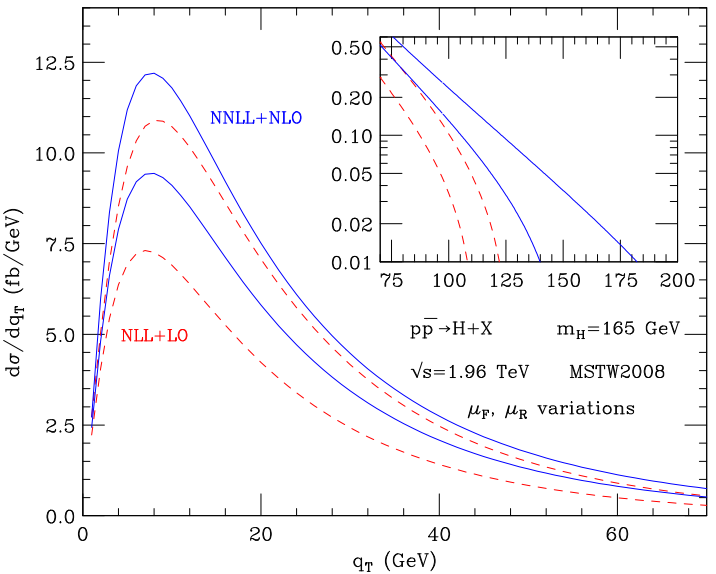

(a)

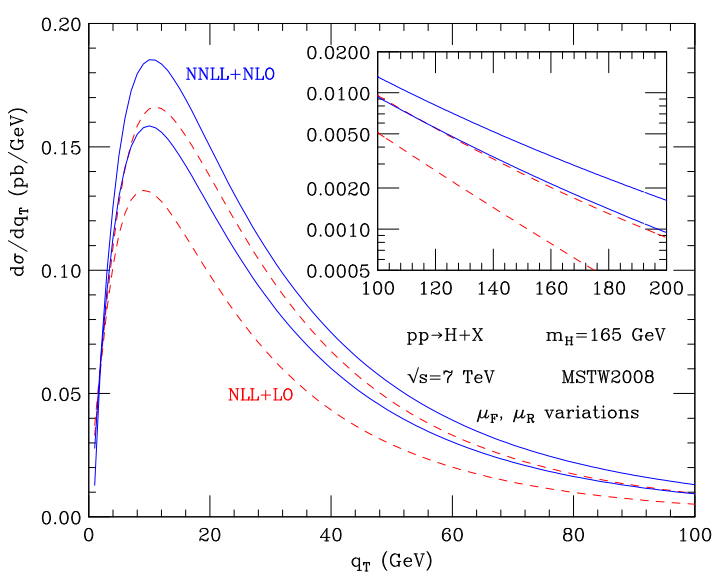

(c)

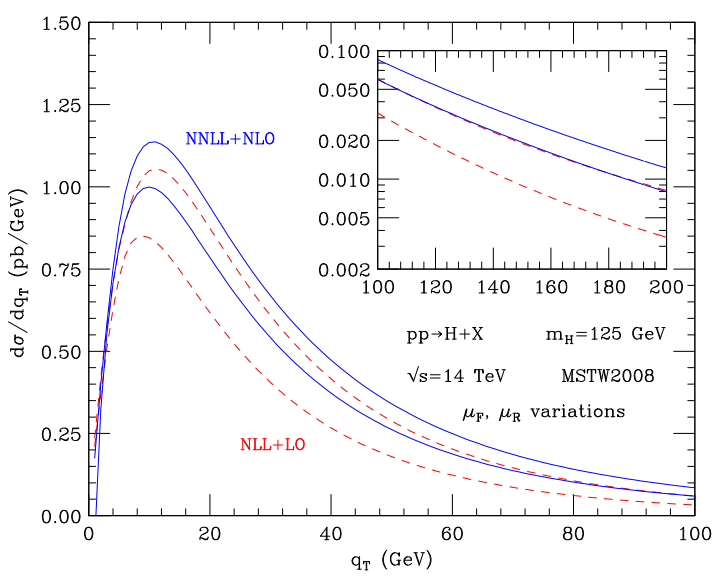

(e)

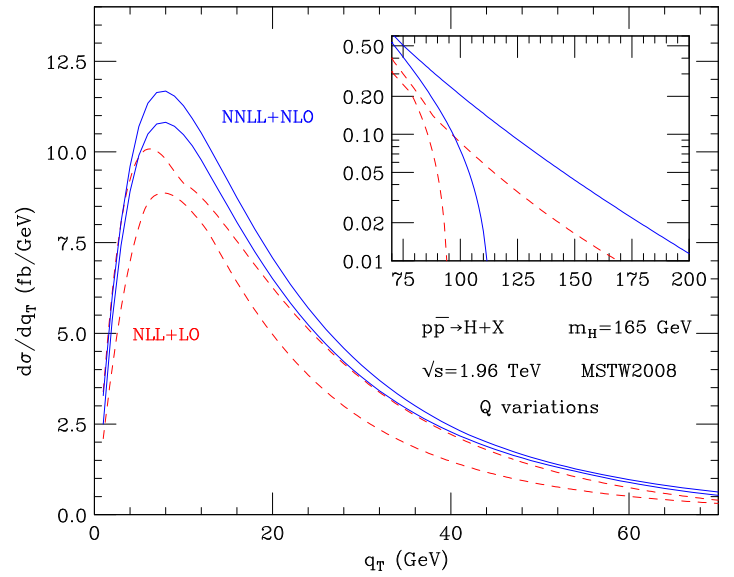

(b)

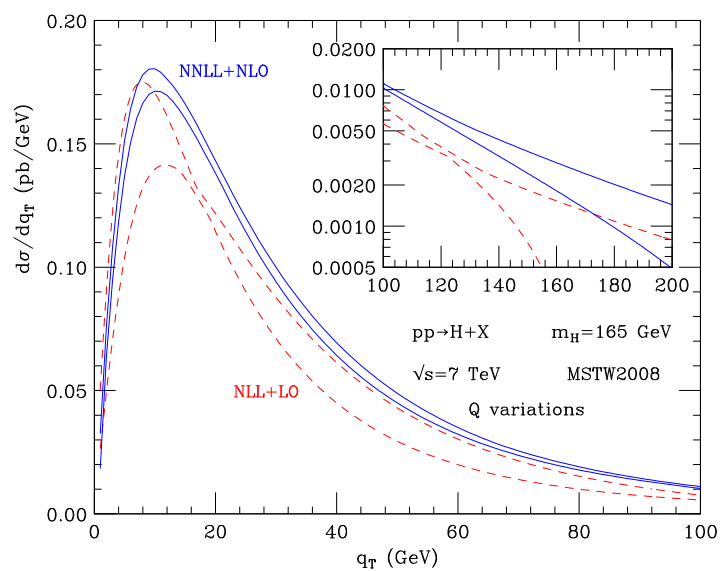

(d)

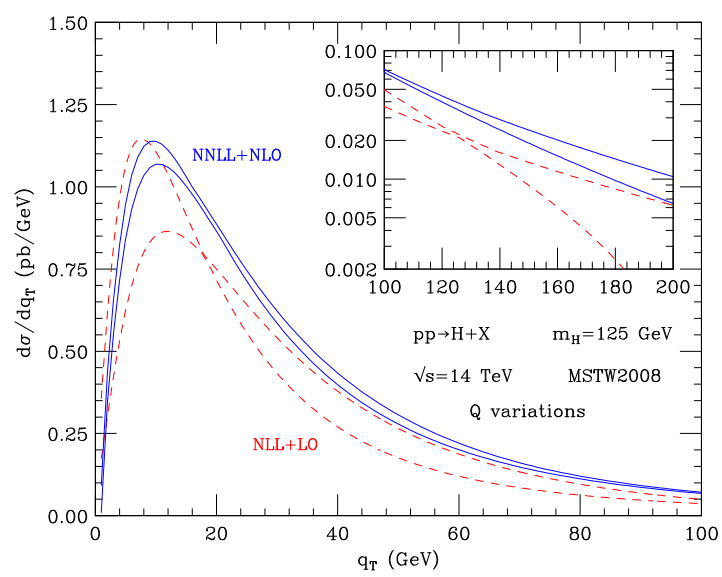

(f)

Figure 2. The $q_{T}$ spectrum of Higgs bosons at the Tevatron and the LHC. The bands are obtained by varying $\mu_{F}$ and $\mu_{R}$ (left panels) and $Q$ (right panels) as described in the text. 
The integral over $q_{T}$ of the resummed NNLL+NLO (NLL+LO) spectrum is in agreement (for any values of $\mu_{R}, \mu_{F}$ and $Q$ ) with the value of the corresponding NNLO (NLO) total cross section to better than $1 \%$, thus checking the numerical accuracy of the code. We also note that the large- $q_{T}$ region gives a little contribution to the total cross section; therefore, the total cross section constraint mainly acts as a perturbative constraint on the resummed spectrum in the region from intermediate to small values of $q_{T}$.

In figure 3 (left panels) we report our NLL+LO and NNLL+NLO total scale uncertainty bands (the inset plots show the large- $q_{T}$ region). The bands represent our best estimate of the perturbative uncertainty, and they are obtained by performing scale variations as follows. We independently vary $\mu_{F}, \mu_{R}$ and $Q$ in the ranges $m_{H} / 2 \leq\left\{\mu_{F}, \mu_{R}\right\} \leq 2 m_{H}$ and $m_{H} / 4 \leq Q \leq m_{H}$, with the constraints $0.5 \leq \mu_{F} / \mu_{R} \leq 2$ and $0.5 \leq Q / \mu_{R} \leq 2$. The constraint on the ratio $\mu_{F} / \mu_{R}$ is the same as used in figure 2 ; it has the purpose of avoiding large logarithmic contributions (powers of $\ln \left(\mu_{F}^{2} / \mu_{R}^{2}\right)$ ) that arise from the evolution of the parton densities. Analogously, the constraint on the ratio $Q / \mu_{R}$ avoids large logarithmic contributions (powers of $\left.\ln \left(Q^{2} / \mu_{R}^{2}\right)\right)$ in the perturbative expansion of the resummed form factor $^{7} \exp \left\{\mathcal{G}_{N}\right\}$ (see eq. (2.6)). We remind the reader (see e.g. eq. (19) of ref. [35]) that the exponent $\mathcal{G}_{N}$ of the form factor is obtained by $q^{2}$ integration of perturbative functions of $\alpha_{\mathrm{S}}\left(q^{2}\right)$ over the range $b_{0}^{2} / b^{2} \leq q^{2} \leq Q^{2}$. To carry out the integration with systematic logarithmic accuracy, the running coupling $\alpha_{\mathrm{S}}\left(q^{2}\right)$ is then expressed in terms of $\alpha_{\mathrm{S}}\left(\mu_{R}\right)$ (and $\left.\ln \left(q^{2} / \mu_{R}^{2}\right)\right)$. As a consequence, the renormalization scale $\mu_{R}$ should not be too different from the resummation scale $Q$, which sets the upper bound of the $q^{2}$ integration.

A more effective way to show the perturbative uncertainties is to consider the fractional difference with respect to a 'reference' central prediction. We choose the NNLL+NLO result at central value of the scales as 'reference' result, $X_{C}$, and we show the ratio $\left(X-X_{C}\right) / X_{C}$ in figure 3 (right panels). The label $X$ refers to the NNLL+NLO results including scale variations (solid lines), and to the NLO results including scale variations (dashed lines).

We comment on the overall perturbative uncertainty band of our results in figure 3 starting from the Tevatron. The NNLL + NLO (NLL+LO) uncertainty is about $\pm 13 \%$ $( \pm 28 \%)$ at the peak, it decreases to about $\pm 10 \%( \pm 23 \%)$ in the region up to $q_{T}=30 \mathrm{GeV}$, and becomes $\pm 18 \%( \pm 20 \%)$ at $q_{T}=60 \mathrm{GeV}$. In the region beyond $q_{T} \sim 80 \mathrm{GeV}$ the resummed result looses predictivity, and its perturbative uncertainty becomes large.

In figure 3(b) the scale variation band of the NLO result is compared to the NNLL+NLO band. The NLO band is obtained by varying $\mu_{F}$ and $\mu_{R}$ as for the NNLL+NLO calculation (the NLO calculation does not depend on the resummation scale $Q$ ). We see that at large values of $q_{T}$ the NLO and NNLL+NLO bands overlap, and the NLO result has smaller uncertainty. As $q_{T}$ becomes smaller than about $80 \mathrm{GeV}$, the NNLL+NLO has a smaller uncertainty, and the bands marginally overlap. In this region of transverse momenta, the effect of resummation starts to set in. When $q_{T}$ becomes smaller and smaller, the NLO band quickly deviates from the NNLL+NLO band and the NLO result becomes unreliable.

We now consider the perturbative uncertainty at the LHC, $\sqrt{s}=7 \mathrm{TeV}$. The NNLL

\footnotetext{
${ }^{7}$ We do not apply additional constraints on the ratio $Q / \mu_{F}$, since the form factor is independent of $\mu_{F}$.
} 


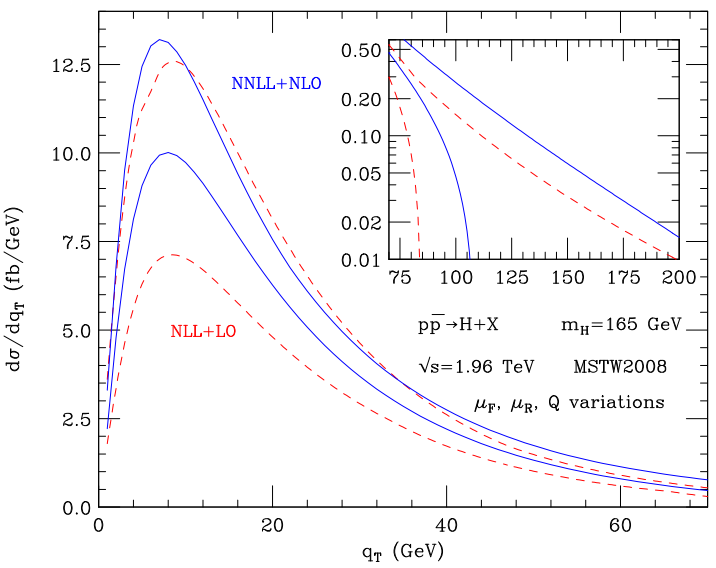

(a)

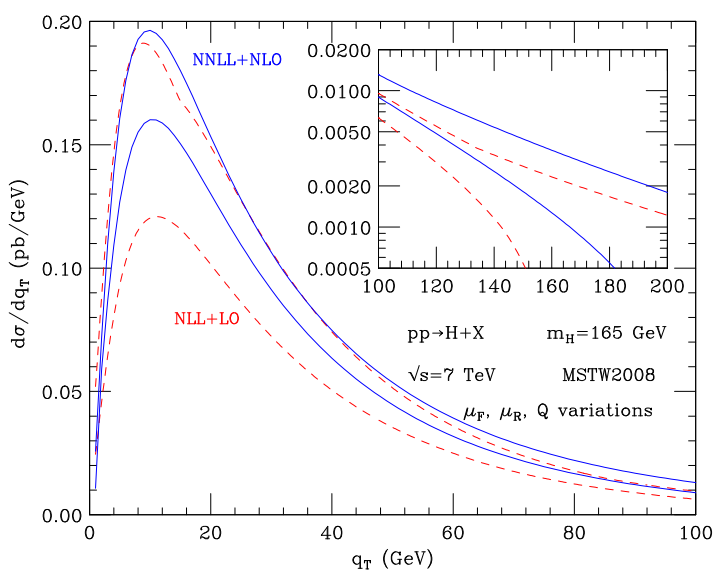

(c)

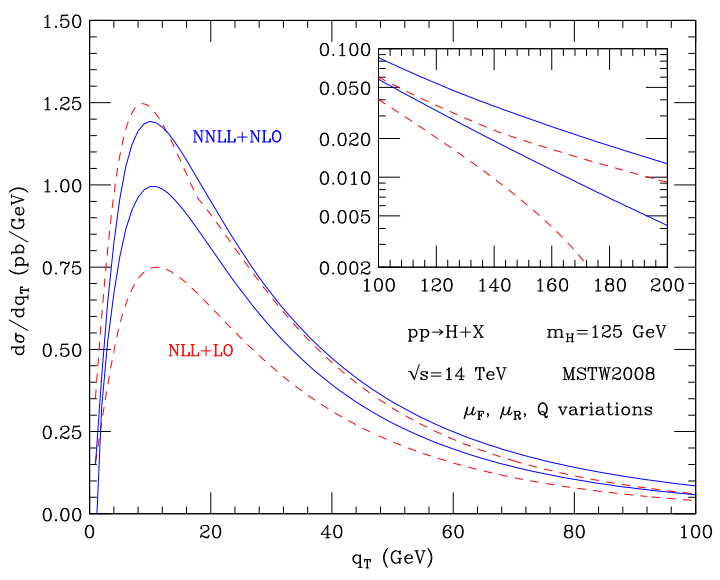

(e)

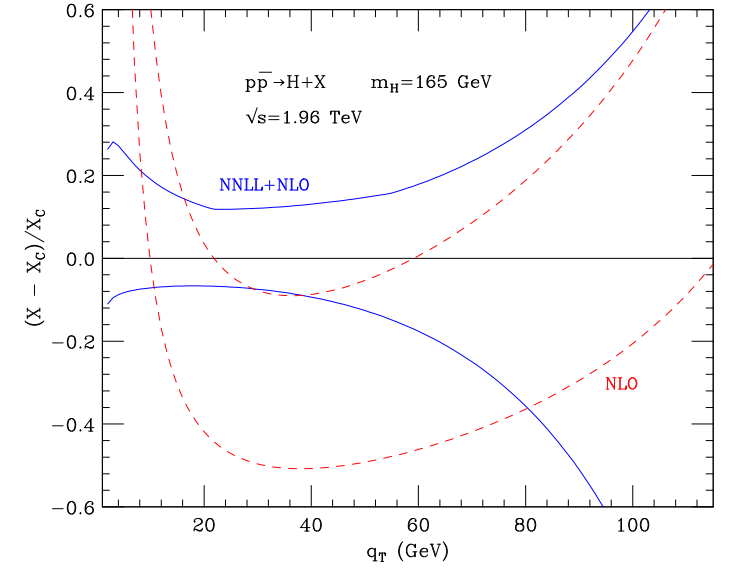

(b)

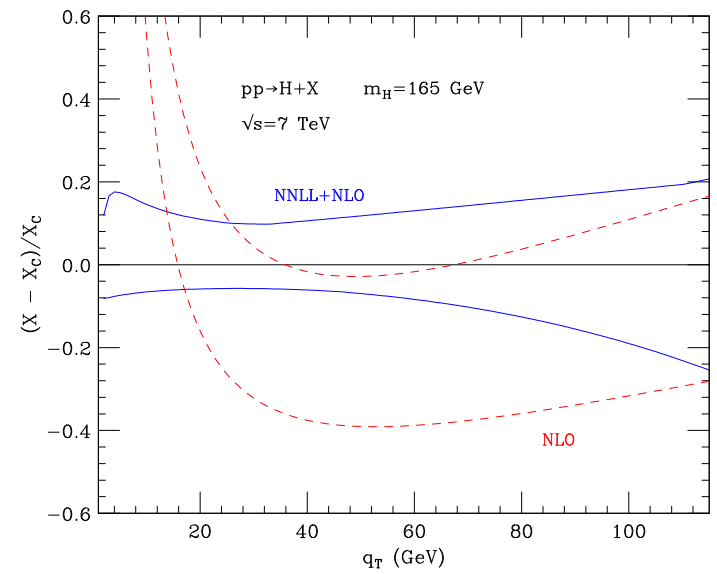

(d)

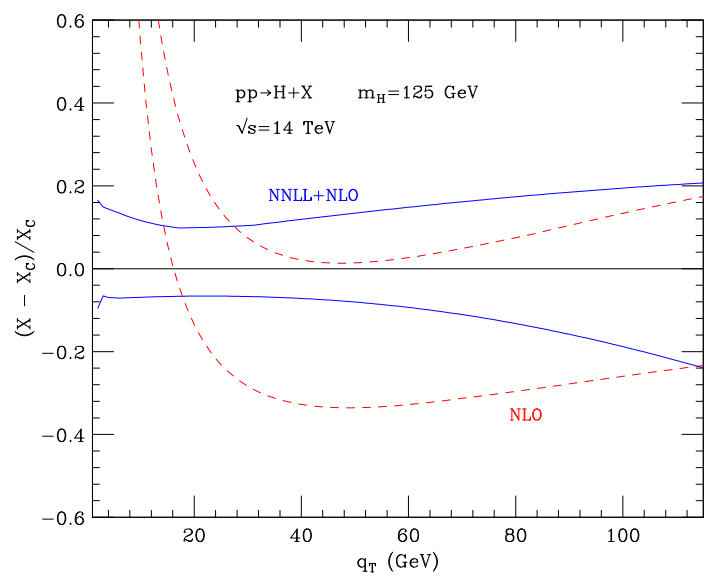

(f)

Figure 3. The $q_{T}$ spectrum of Higgs bosons at the Tevatron and the LHC: NNLL+NLO (solid) and NNL+LO (dashes) uncertainty bands (left panels); NNLL+NLO (solid) and NLO (dashes) uncertainty bands relative to the central NNLL+NLO result (right panels). 
$+\mathrm{NLO}(\mathrm{NLL}+\mathrm{LO})$ uncertainty is about $\pm 10 \%( \pm 22 \%)$ at the peak, it decreases to about $\pm 8 \%( \pm 19 \%)$ in the region up to $q_{T}=30 \mathrm{GeV}$, and becomes $\pm 10 \%( \pm 18 \%)$ at $q_{T}=60 \mathrm{GeV}$. In the region beyond $q_{T} \sim 120 \mathrm{GeV}$ the resummed result looses predictivity, and its perturbative uncertainty becomes large. In figure 3(d) we compare the NLO and NNLL+NLO bands. The qualitative features are similar to figure 3(b): at large values of $q_{T}$ the NLO and NNLL+NLO scale uncertainty bands overlap, and the NLO result has smaller uncertainty. As $q_{T}$ becomes smaller than about $120 \mathrm{GeV}$, the NNLL+NLO has a smaller uncertainty, but the bands still overlap. In the region of intermediate transverse momenta $\left(q_{T} \sim 50 \mathrm{GeV}\right)$, the bands marginally overlap and the NLO result underestimates the cross section. When $q_{T}$ becomes smaller, the NLO band quickly deviates from the NNLL+NLO band and the NLO result becomes unreliable.

We finally consider the perturbative uncertainty at the LHC when $\sqrt{s}=14 \mathrm{TeV}$. The NNLL + NLO (NLL+LO) uncertainty is about $\pm 9 \%( \pm 25 \%)$ at the peak, it decreases to about $\pm 8 \%( \pm 19 \%)$ in the region up to $q_{T}=30 \mathrm{GeV}$, and moves to $\pm 12 \%( \pm 19 \%)$ at $q_{T}=60 \mathrm{GeV}$. In the region beyond $q_{T} \sim 150 \mathrm{GeV}$ the resummed result looses predictivity, and its perturbative uncertainty becomes large. In figure $3(\mathrm{f})$ we compare the NLO and NNLL+NLO scale uncertainty bands. The qualitative features are similar to those of figures 3(b), 3(d): at large values of $q_{T}$ the NLO and NNLL+NLO bands overlap and the NLO result has smaller uncertainty. In the region of intermediate transverse momenta $\left(q_{T} \sim 50 \mathrm{GeV}\right)$, the bands marginally overlap and the NLO result underestimates the cross section. When $q_{T}$ becomes smaller, the NLO result becomes unreliable.

Comparing figure 3(a), 3(b) with figure 3(c), 3(d) and figure 3(e), 3(f) we see that perturbative uncertainties are larger at the Tevatron than at the LHC. We also note that our NNLL+NLO result is much more stable at the LHC than at the Tevatron, where its validity is confined to a smaller region of transverse momenta. This is not completely unexpected. At smaller values of the center of mass energy, the production of the Higgs boson is accompanied by softer radiation, and thus the $q_{T}$ spectrum is softer than at the LHC.

We finally discuss the uncertainties on the normalized $q_{T}$ spectrum (i.e., $1 / \sigma \times d \sigma / d q_{T}$ ). As mentioned in the introduction, the typical procedure of the experimental collaborations is to use the information on the total cross section [18] to rescale the best theoretical predictions of Monte Carlo event generators, whereas the NNLL+NLO result of our calculation, obtained with the public program HqT, is used to reweight the transverse-momentum spectrum of the Higgs boson obtained in the simulation. Such a procedure implies that the important information provided by the resummed NNLL+NLO spectrum is not its integral, i.e. the total cross section, but its shape. The sources of uncertainties on the shape of the spectrum are essentially the same as for the inclusive cross section: the uncertainty from missing higher-order contributions, estimated through scale variations, PDF uncertainties, and the uncertainty from the use of the large- $M_{t}$ approximation, that we discussed at the beginning of this section. One additional uncertainty in the $q_{T}$ spectrum that needs be considered comes from Non-Perturbative (NP) effects.

We remind the reader that the quantitative predictions presented in this paper are obtained in a purely perturbative framework. It is known [23-32] that the transversemomentum distribution is affected by NP effects, which become important as $q_{T}$ becomes 
small. A customary way of modelling these effects is to introduce an NP transversemomentum smearing of the distribution. In the case of resummed calculations in impact parameter space, the NP smearing is implemented by multiplying the $b$-space perturbative form factor by an NP form factor. The parameters controlling this NP form factor are typically obtained through a comparison to data. Since there is no evidence for the Higgs boson yet, the procedure to fix the NP form factor is somewhat arbitrary. Here we follow the procedure adopted in ref. [35], and we multiply the resummed form factor in eq. (2.4) by a gaussian smearing $S_{N P}=\exp \left\{-g b^{2}\right\}$, where the parameter $g$ is taken in the range $\left(g=1.67-5.64 \mathrm{GeV}^{2}\right)$ suggested by the study of ref. $[60] .{ }^{8}$ The above procedure can give us some insight on the quantitative impact of these NP effects on the Higgs boson spectrum.

In figure 4 (left panels) we compare the NNLL+NLO shape uncertainty as coming from scale variations (solid lines) to the NP effects (dashed lines). The bands are obtained by normalizing each spectrum to unity, and computing the relative difference with respect to the central normalized prediction obtained with the MSTW2008 NNLO set (with $g=$ 0). A comparison of figure 4(a), 4(c), 4(e) to figure 3(b), 3(d), 3(f) shows that the scale uncertainty on the normalized NNLL+NLO distribution is smaller than the corresponding uncertainty on the NNLL+NLO result. This is not unexpected: a sizeable contribution to the uncertainties shown in figure 3 comes actually from uncertainties on the total cross section, which do not contribute in figure 4. In other words, studying uncertainties on the normalized distribution allows us to assess the true uncertainty in the shape of the resummed $q_{T}$ spectrum.

At the Tevatron (figure 4(a)) such scale uncertainty ranges from $+8 \%-3 \%$ in the region of the peak, to $+3 \%-8 \%$ when $q_{T} \sim 50 \mathrm{GeV}$. At larger values of $q_{T}$ the uncertainty of the NNLL+NLO resummed distribution increases consistently with the behaviour observed in figure 3(b). The inclusion of the NP effects makes the distribution harder, the effect ranging from $10 \%$ to $20 \%$ in the very small- $q_{T}$ region. For $q_{T} \gtrsim 10 \mathrm{GeV}$ the impact of NP effects is of the order of about $5 \%$ and decreases as $q_{T}$ increases. At the LHC, $\sqrt{s}=7 \mathrm{TeV}$ (figure $4(\mathrm{c})$ ) the scale uncertainty ranges from $+5 \%-3 \%$ in the region of the peak to $+5 \%-4 \%$ at $q_{T} \sim 80 \mathrm{GeV}$. At the LHC, $\sqrt{s}=14 \mathrm{TeV}$ (figure $4(\mathrm{e})$ ) the shape uncertainty ranges from $+5 \%-3 \%$ in the region of the peak to $+8 \%-9 \%$ at $q_{T} \sim 100 \mathrm{GeV}$. The impact of NP effects is similar at $\sqrt{s}=7$ and $14 \mathrm{TeV}$ : it ranges from about $10 \%$ to $20 \%$ in the region below the peak, is about $3-4 \%$ for $q_{T} \sim 20 \mathrm{GeV}$, and quickly decreases as $q_{T}$ increases. We conclude that the uncertainty from unknown NP effects is smaller than the scale uncertainty, and is comparable to the latter only in the very small $q_{T}$ region.

The impact of PDF uncertainties at $68 \% \mathrm{CL}$ on the shape of the $q_{T}$ spectrum is studied in figures 4(b), 4(d), 4(f). By evaluating PDF uncertainties with MSTW2008 NNLO PDFs (red band in figures 4(b), 4(d), 4(f)) we see that the uncertainty is at the $\pm 1-2 \%$ level, both at the Tevatron and at the LHC. The use of different PDF sets affects not only the absolute value of the NNLO cross section (see e.g. ref. [82]) but also the shape of the $q_{T}$ spectrum. The predictions obtained with NNPDF 2.1 PDFs are in good

\footnotetext{
${ }^{8}$ We note that the inclusion of this smearing factor does not change the overall normalization, since $S_{N P}(b=0)=1$
} 
agreement with those obtained with the MSTW2008 set and the uncertainty bands overlap over a wide range of transverse momenta. On the contrary, the prediction obtained with the ABKM09 NNLO set is softer and the uncertainty band does not overlap with the MSTW2008 band. This behaviour is not completely unexpected: when the Higgs boson is produced at large transverse momenta, larger values of Bjorken $x$ are probed, where the ABKM gluon is smaller than MSTW2008 one. The JR09 band shows a good compatibility with the MSTW2008 result, at least at the Tevatron and at the LHC for $\sqrt{s}=7 \mathrm{TeV}$, where the uncertainty is however rather large. At the LHC for $\sqrt{s}=14 \mathrm{TeV}$ the differences with the MSTW2008 result are more pronounced.

We conclude this section by adding few comments on the work of ref. [62]. In this paper the RESBOS generator $[56,57]$, which is based on the classical $b$-space resummation formalism [23-32], is used to perform a new computation of the Higgs $q_{T}$ spectrum at the Tevatron and the LHC. The NNLL resummed calculation in the low $q_{T}$ region is matched to the NLO result at high $q_{T}[21]$. Besides the differences in the resummation formalism (see ref. [35] for a detailed discussion) there are few differences with respect to the work presented here. Our calculation implements the coefficient $A^{(3)}$ from ref. [54], whereas in ref. [62] the authors implement the result of ref. [72] for threshold resummation. The calculation of ref. [62] does not exploit a unitarity constraint on the total cross section, and thus the normalization of the ensuing resummed spectra is not fully defined. Finally, the calculation of ref. [62] does not include the hard collinear coefficients $\mathcal{H}^{H(2)}$. This produces sizeable quantitative differences with our results in the low $q_{T}$ region. Comparing our NNLL+NLO results with those of ref. [62] we find that the height of the peak of our spectra is typically higher, by about $20-30 \%$, mainly due to the (positive) impact of $\mathcal{H}^{H(2)}$.

\section{Summary}

In this paper we have considered the $q_{T}$ spectrum of Higgs bosons produced in hadron collisions, and we have presented a perturbative QCD study based on transverse-momentum resummation up to NNLL+NLO accuracy.

We have followed the formalism developed in refs. [34-36], which is valid for the production of a generic high-mass system of non strongly-interacting particles in hadron collisions. The formalism combines small- $q_{T}$ resummation at a given logarithmic accuracy with the fixed-order calculations. It implements a unitarity constraint that guarantees that the integral over $q_{T}$ of the differential cross section coincides with the total cross section at the corresponding fixed-order accuracy. This leads to QCD predictions with a controllable and uniform perturbative accuracy over the region from small up to large values of $q_{T}$. At large values of $q_{T}$, the resummation formalism is superseded by customary fixed-order calculations.

We have considered Higgs bosons produced by gluon fusion in $p \bar{p}$ collisions at the Tevatron and $p p$ collisions at LHC energies, and we have presented an update of the phenomenological analysis of ref. [35]. The calculation now includes the exact value of the NNLO hard-collinear coefficients $\mathcal{H}_{N}^{H(2)}$ computed in ref. [52, 53], and the recently derived value of the NNLL coefficient $A^{(3)}$ [54]. 


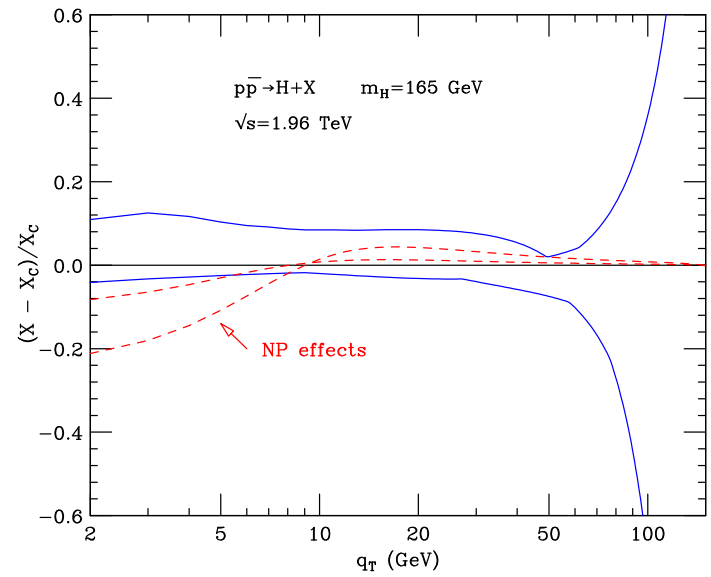

(a)

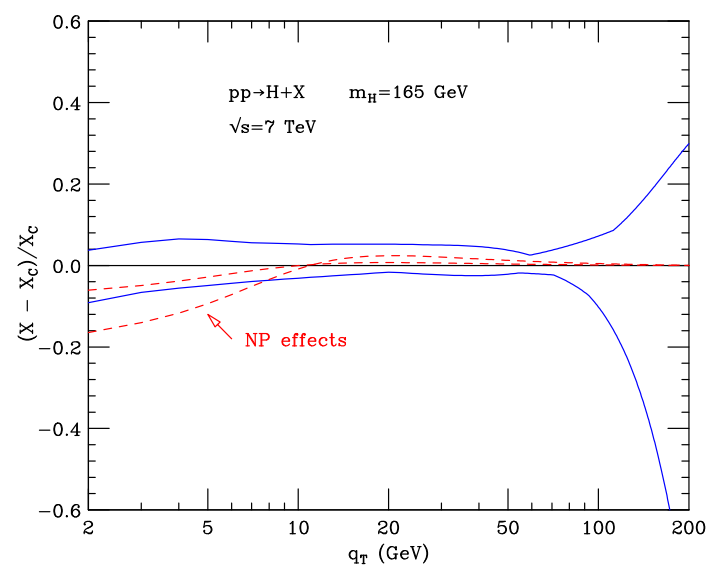

(c)

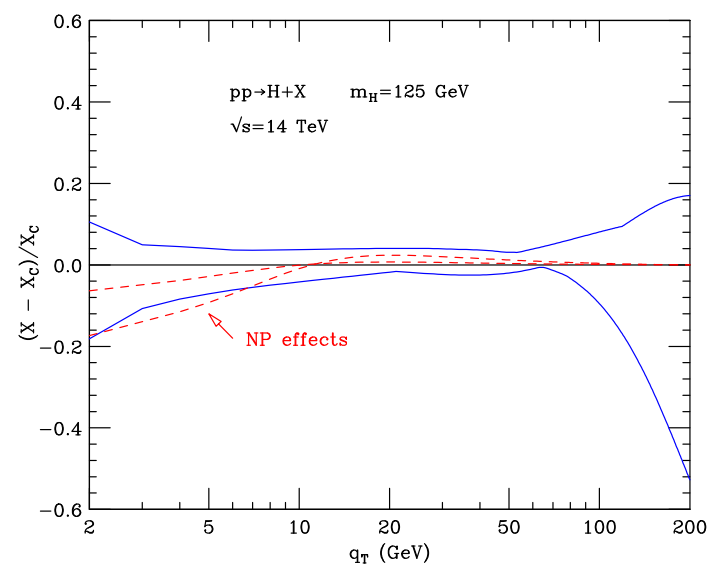

(e)

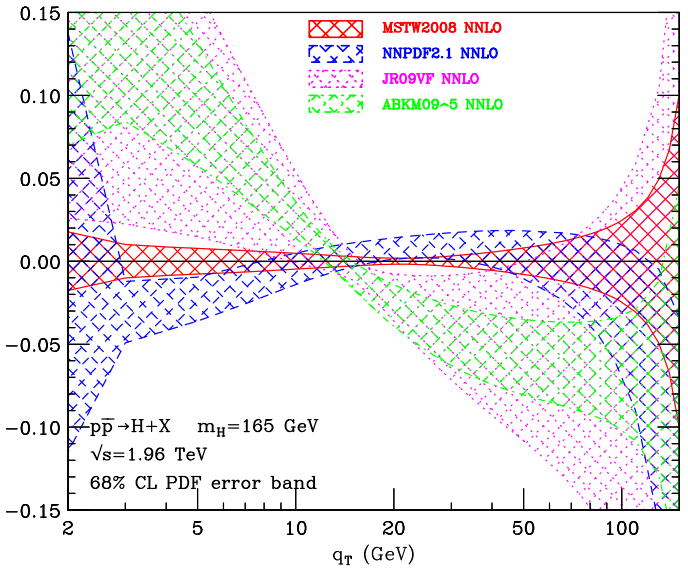

(b)

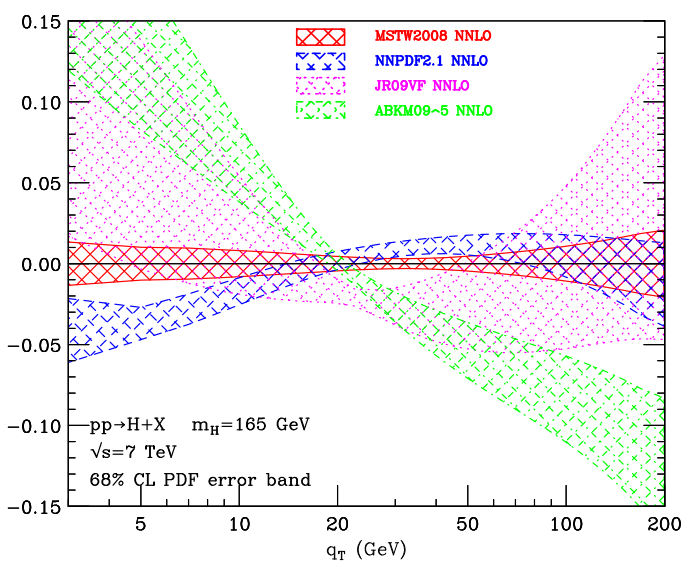

(d)

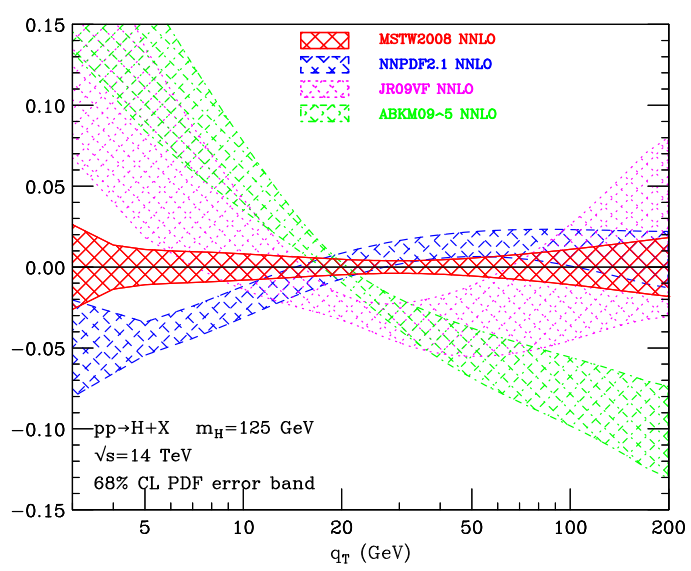

(f)

Figure 4. Uncertainties in the normalized $q_{T}$ spectrum of the Higgs boson at the Tevatron and the LHC. Left panels: the NNLL+NLO uncertainty bands (solid) computed as in figure 3 compared to an estimate of NP effects (dashed). Right panels: PDF uncertainties bands at 68\% CL. All results are relative to the NNLL+NLO central value computed with MSTW2008 NNLO PDFs. 
We have performed a study of the scale dependence of our results to estimate the corresponding perturbative uncertainty. In a wide region of transverse momenta the size of the scale uncertainties is considerably reduced in going from NLL+LO to NNLL+NLO accuracy.

Our calculation for the $q_{T}$ spectrum is implemented in the updated version of the numerical code HqT. We have argued that, given the use that is currently done of our numerical program, the important information is in the shape of the $q_{T}$ spectrum. We have thus studied the uncertainties of the normalized spectrum, comparing scale and PDF uncertainties, and estimating the impact of NP effects.

\section{Acknowledgments}

This work has been supported in part by the European Commission through the 'LHCPhenoNet' Initial Training Network PITN-GA-2010-264564.

\section{References}

[1] LeP Working Group for Higgs boson searches, ALEPH, DELPHI, L3, OPAL collaboration, R. Barate et al., Search for the standard model Higgs boson at LEP, Phys. Lett. B 565 (2003) 61 [hep-ex/0306033] [INSPIRE].

[2] CDF and D0 collaboration, Combined CDF and D0 upper limits on standard model Higgs boson production with up to $8.6 \mathrm{fb}^{-1}$ of data, arXiv:1107.5518 [INSPIRE].

[3] ATLAS collaboration, Combination of the searches for the Higgs boson in $\sim 1 \mathrm{fb}^{-1}$ of data taken with the ATLAS detector at $7 \mathrm{TeV}$ center-of-mass energy, ATLAS-CONF-2011-112 (2011).

[4] CMS collaboration, SM Higgs combination, CMS-HIG-11-011 (2011).

[5] ATLAS collaboration, Update of the combination of Higgs boson searches in pp collisions at sqrt $(s)=7 \mathrm{TeV}$ with the ATLAS Experiment at the LHC, ATLAS-CONF-2011-135 (2011).

[6] CMS collaboration, Combination of Higgs Searches, PAS-HIG-11-022 (2011).

[7] C. Anastasiou, R. Boughezal and F. Petriello, Mixed QCD-electroweak corrections to Higgs boson production in gluon fusion, JHEP 04 (2009) 003 [arXiv: 0811.3458] [INSPIRE].

[8] D. de Florian and M. Grazzini, Higgs production through gluon fusion: updated cross sections at the Tevatron and the LHC, Phys. Lett. B 674 (2009) 291 [arXiv:0901.2427] [INSPIRE].

[9] R.V. Harlander and W.B. Kilgore, Next-to-next-to-leading order Higgs production at hadron colliders, Phys. Rev. Lett. 88 (2002) 201801 [hep-ph/0201206] [INSPIRE].

[10] C. Anastasiou and K. Melnikov, Higgs boson production at hadron colliders in NNLO QCD, Nucl. Phys. B 646 (2002) 220 [hep-ph/0207004] [InSPIRE].

[11] V. Ravindran, J. Smith and W.L. van Neerven, NNLO corrections to the total cross-section for Higgs boson production in hadron hadron collisions, Nucl. Phys. B 665 (2003) 325 [hep-ph/0302135] [INSPIRE].

[12] S. Catani, D. de Florian, M. Grazzini and P. Nason, Soft gluon resummation for Higgs boson production at hadron colliders, JHEP 07 (2003) 028 [hep-ph/0306211] [INSPIRE].

[13] U. Aglietti, R. Bonciani, G. Degrassi and A. Vicini, Two loop light fermion contribution to Higgs production and decays, Phys. Lett. B 595 (2004) 432 [hep-ph/0404071] [inSPIRE]. 
[14] G. Degrassi and F. Maltoni, Two-loop electroweak corrections to Higgs production at hadron colliders, Phys. Lett. B 600 (2004) 255 [hep-ph/0407249] [INSPIRE].

[15] U. Aglietti, R. Bonciani, G. Degrassi and A. Vicini, Two-loop electroweak corrections to Higgs production in proton-proton collisions, hep-ph/0610033 [INSPIRE].

[16] S. Actis, G. Passarino, C. Sturm and S. Uccirati, NLO electroweak corrections to Higgs boson production at hadron colliders, Phys. Lett. B 670 (2008) 12 [arXiv:0809.1301] [INSPIRE].

[17] S. Actis, G. Passarino, C. Sturm and S. Uccirati, NNLO computational techniques: the cases $H \rightarrow \gamma \gamma$ and $H \rightarrow g g$, Nucl. Phys. B 811 (2009) 182 [arXiv:0809.3667] [InSPIRE].

[18] LhC Higgs Cross Section Working Group collaboration, S. Dittmaier et al., Handbook of LHC Higgs Cross Sections: 1. Inclusive Observables, arXiv:1101.0593 [INSPIRE].

[19] D. de Florian, M. Grazzini and Z. Kunszt, Higgs production with large transverse momentum in hadronic collisions at next-to-leading order, Phys. Rev. Lett. 82 (1999) 5209 [hep-ph/9902483] [INSPIRE].

[20] V. Ravindran, J. Smith and W. Van Neerven, Next-to-leading order QCD corrections to differential distributions of Higgs boson production in hadron hadron collisions, Nucl. Phys. B 634 (2002) 247 [hep-ph/0201114] [INSPIRE].

[21] C.J. Glosser and C.R. Schmidt, Next-to-leading corrections to the Higgs boson transverse momentum spectrum in gluon fusion, JHEP 12 (2002) 016 [hep-ph/0209248] [INSPIRE].

[22] D. de Florian, A. Kulesza and W. Vogelsang, Threshold resummation for high-transverse-momentum Higgs production at the LHC, JHEP 02 (2006) 047 [hep-ph/0511205] [INSPIRE].

[23] Y.L. Dokshitzer, D. Diakonov and S. Troian, On the transverse momentum distribution of massive lepton pairs, Phys. Lett. B 79 (1978) 269 [InSPIRE].

[24] Y.L. Dokshitzer, D. Diakonov and S. Troian, Hard processes in quantum chromodynamics, Phys. Rept. 58 (1980) 269 [INSPIRE].

[25] G. Parisi and R. Petronzio, Small transverse momentum distributions in hard processes, Nucl. Phys. B 154 (1979) 427 [InSPIRE].

[26] G. Curci, M. Greco and Y. Srivastava, QCD jets from coherent states, Nucl. Phys. B 159 (1979) 451 [InSPIRE].

[27] J.C. Collins and D.E. Soper, Back-to-back jets in QCD, Nucl. Phys. B 193 (1981) 381 [Erratum ibid. B 213 (1983) 545] [INSPIRE].

[28] J.C. Collins and D.E. Soper, Back-to-back jets: Fourier transform from B to K-transverse, Nucl. Phys. B 197 (1982) 446 [inSPIRE].

[29] J.C. Collins, D.E. Soper and G.F. Sterman, Transverse momentum distribution in Drell-Yan pair and $W$ and $Z$ boson production, Nucl. Phys. B 250 (1985) 199 [INSPIRE].

[30] J. Kodaira and L. Trentadue, Summing soft emission in QCD, Phys. Lett. B 112 (1982) 66 [INSPIRE].

[31] J. Kodaira and L. Trentadue, Soft gluon effects in perturbative quantum chromodynamics, SLAC-PUB-2934 (1982) [INSPIRE].

[32] J. Kodaira and L. Trentadue, Single logarithm effects in electron-positron annihilation, Phys. Lett. B 123 (1983) 335 [INSPIRE].

[33] S. Catani, E. D'Emilio and L. Trentadue, The gluon form-factor to higher orders: gluon gluon annihilation at small Q-transverse, Phys. Lett. B 211 (1988) 335 [INSPIRE]. 
[34] S. Catani, D. de Florian and M. Grazzini, Universality of nonleading logarithmic contributions in transverse momentum distributions, Nucl. Phys. B 596 (2001) 299 [hep-ph/0008184] [INSPIRE].

[35] G. Bozzi, S. Catani, D. de Florian and M. Grazzini, Transverse-momentum resummation and the spectrum of the Higgs boson at the LHC, Nucl. Phys. B 737 (2006) 73 [hep-ph/0508068] [INSPIRE].

[36] S. Catani and M. Grazzini, QCD transverse-momentum resummation in gluon fusion processes, Nucl. Phys. B 845 (2011) 297 [arXiv:1011.3918] [INSPIRE].

[37] S. Frixione and B.R. Webber, Matching NLO QCD computations and parton shower simulations, JHEP 06 (2002) 029 [hep-ph/0204244] [INSPIRE].

[38] P. Nason, A new method for combining NLO QCD with shower Monte Carlo algorithms, JHEP 11 (2004) 040 [hep-ph/0409146] [INSPIRE].

[39] S. Frixione, P. Nason and C. Oleari, Matching NLO QCD computations with parton shower simulations: the POWHEG method, JHEP 11 (2007) 070 [arXiv:0709.2092] [INSPIRE].

[40] D. de Florian and M. Grazzini, Next-to-next-to-leading logarithmic corrections at small transverse momentum in hadronic collisions, Phys. Rev. Lett. 85 (2000) 4678 [hep-ph/0008152] [INSPIRE].

[41] G. Bozzi, S. Catani, D. de Florian and M. Grazzini, The $q(T)$ spectrum of the Higgs boson at the LHC in QCD perturbation theory, Phys. Lett. B 564 (2003) 65 [hep-ph/0302104] [INSPIRE].

[42] G. Bozzi, S. Catani, D. de Florian and M. Grazzini, Higgs boson production at the LHC: transverse-momentum resummation and rapidity dependence, Nucl. Phys. B 791 (2008) 1 [arXiv: 0705.3887] [INSPIRE].

[43] G. Bozzi, S. Catani, G. Ferrera, D. de Florian and M. Grazzini, Transverse-momentum resummation: a perturbative study of $Z$ production at the Tevatron,

Nucl. Phys. B 815 (2009) 174 [arXiv:0812.2862] [INSPIRE].

[44] G. Bozzi, S. Catani, G. Ferrera, D. de Florian and M. Grazzini, Production of Drell-Yan lepton pairs in hadron collisions: transverse-momentum resummation at next-to-next-to-leading logarithmic accuracy, Phys. Lett. B 696 (2011) 207 [arXiv: 1007.2351] [INSPIRE].

[45] M. Grazzini, Soft-gluon effects in WW production at hadron colliders, JHEP 01 (2006) 095 [hep-ph/0510337] [INSPIRE].

[46] R. Frederix and M. Grazzini, Higher-order QCD effects in the $h \rightarrow Z Z$ search channel at the LHC, Phys. Lett. B 662 (2008) 353 [arXiv:0801.2229] [INSPIRE].

[47] G. Bozzi, B. Fuks and M. Klasen, Transverse-momentum resummation for slepton-pair production at the CERN LHC, Phys. Rev. D 74 (2006) 015001 [hep-ph/0603074] [INSPIRE].

[48] H. Kawamura, J. Kodaira, H. Shimizu and K. Tanaka, The dilepton $Q(T)$ spectrum in transversely polarized Drell-Yan process in QCD, Prog. Theor. Phys. 115 (2006) 667 [hep-ph/0512137] [INSPIRE].

[49] H. Kawamura, J. Kodaira and K. Tanaka, Soft gluon corrections to double transverse-spin asymmetries for small-Q(T) dilepton production at RHIC and J-PARC, Nucl. Phys. B 777 (2007) 203 [hep-ph/0703079] [INSPIRE].

[50] H. Kawamura, J. Kodaira and K. Tanaka, Transversely polarized Drell-Yan process and soft gluon resummation in QCD, Prog. Theor. Phys. 118 (2007) 581 [arXiv:0709.1752] [INSPIRE]. 
[51] H. Kawamura, J. Kodaira and K. Tanaka, Double-spin asymmetries for small-Q(T) Drell-Yan pair production in transversely polarized p $\bar{p}$ collisions, Phys. Lett. B 662 (2008) 139 [arXiv:0801.0026] [InSPIRE].

[52] S. Catani and M. Grazzini, An NNLO subtraction formalism in hadron collisions and its application to Higgs boson production at the LHC, Phys. Rev. Lett. 98 (2007) 222002 [hep-ph/0703012] [INSPIRE].

[53] S. Catani and M. Grazzini, Higgs boson production at hadron colliders: hard-collinear coefficients at the NNLO, arXiv:1106.4652 [INSPIRE].

[54] T. Becher and M. Neubert, Drell-Yan production at small $q_{T}$, transverse parton distributions and the collinear anomaly, Eur. Phys. J. C 71 (2011) 1665 [arXiv: 1007.4005] [INSPIRE].

[55] M. Grazzini, Higgs production at hadron colliders tools, http://theory.fi.infn.it/grazzini/codes.html.

[56] C. Balázs and C. Yuan, Higgs boson production at the LHC with soft gluon effects, Phys. Lett. B 478 (2000) 192 [hep-ph/0001103] [INSPIRE].

[57] C. Balázs, J. Huston and I. Puljak, Higgs production: a comparison of parton showers and resummation, Phys. Rev. D 63 (2001) 014021 [hep-ph/0002032] [INSPIRE].

[58] E.L. Berger and J.-w. Qiu, Differential cross-section for Higgs boson production including all orders soft gluon resummation, Phys. Rev. D 67 (2003) 034026 [hep-ph/0210135] [INSPIRE].

[59] E.L. Berger and J.-w. Qiu, Differential cross-sections for Higgs boson production at Tevatron collider energies, Phys. Rev. Lett. 91 (2003) 222003 [hep-ph/0304267] [INSPIRE].

[60] A. Kulesza and W.J. Stirling, Nonperturbative effects and the resummed Higgs transverse momentum distribution at the LHC, JHEP 12 (2003) 056 [hep-ph/0307208] [INSPIRE].

[61] A. Kulesza, G.F. Sterman and W. Vogelsang, Joint resummation for Higgs production, Phys. Rev. D 69 (2004) 014012 [hep-ph/0309264] [INSPIRE].

[62] Q.-H. Cao, C.-R. Chen, C. Schmidt and C.-P. Yuan, Improved predictions for Higgs Q(T) at the Tevatron and the LHC, arXiv:0909.2305 [INSPIRE].

[63] S. Mantry and F. Petriello, Transverse momentum distributions from effective field theory with numerical results, Phys. Rev. D 83 (2011) 053007 [arXiv: 1007.3773] [INSPIRE].

[64] R. Kauffman, Higher order corrections to Higgs boson $p_{T}$, Phys. Rev. D 45 (1992) 1512 [INSPIRE].

[65] C. Yuan, Kinematics of the Higgs boson at hadron colliders: NLO QCD gluon resummation, Phys. Lett. B 283 (1992) 395 [INSPIRE].

[66] J. Blumlein, Analytic continuation of Mellin transforms up to two loop order, Comput. Phys. Commun. 133 (2000) 76 [hep-ph/0003100] [INSPIRE].

[67] J. Blumlein and S. Kurth, Harmonic sums and Mellin transforms up to two loop order, Phys. Rev. D 60 (1999) 014018 [hep-ph/9810241] [INSPIRE].

[68] J. Blumlein and V. Ravindran, Mellin moments of the next-to-next-to leading order coefficient functions for the Drell-Yan process and hadronic Higgs-boson production, Nucl. Phys. B 716 (2005) 128 [hep-ph/0501178] [INSPIRE].

[69] J. Blumlein and S.-O. Moch, Analytic continuation of the harmonic sums for the 3-loop anomalous dimensions, Phys. Lett. B 614 (2005) 53 [hep-ph/0503188] [INSPIRE].

[70] E. Laenen, G.F. Sterman and W. Vogelsang, Higher order QCD corrections in prompt photon production, Phys. Rev. Lett. 84 (2000) 4296 [hep-ph/0002078] [INSPIRE]. 
[71] A. Kulesza, G.F. Sterman and W. Vogelsang, Joint resummation in electroweak boson production, Phys. Rev. D 66 (2002) 014011 [hep-ph/0202251] [INSPIRE].

[72] S. Moch, J. Vermaseren and A. Vogt, The three loop splitting functions in QCD: the nonsinglet case, Nucl. Phys. B 688 (2004) 101 [hep-ph/0403192] [INSPIRE].

[73] U. Baur and E. Glover, Higgs boson production at large transverse momentum in hadronic collisions, Nucl. Phys. B 339 (1990) 38 [INSPIRE].

[74] H.-L. Lai et al., New parton distributions for collider physics, Phys. Rev. D 82 (2010) 074024 [arXiv:1007.2241] [INSPIRE].

[75] R.D. Ball et al., A first unbiased global NLO determination of parton distributions and their uncertainties, Nucl. Phys. B 838 (2010) 136 [arXiv: 1002.4407] [INSPIRE].

[76] H1 collaboration, F. Aaron et al., A precision measurement of the inclusive ep scattering cross section at HERA, Eur. Phys. J. C 64 (2009) 561 [arXiv:0904.3513] [InSPIRE].

[77] A. Martin, W. Stirling, R. Thorne and G. Watt, Parton distributions for the LHC, Eur. Phys. J. C 63 (2009) 189 [arXiv:0901.0002] [InSPIRE].

[78] S. Alekhin, J. Blumlein, S. Klein and S. Moch, The 3, 4 and 5-flavor NNLO parton from deep-inelastic-scattering data and at hadron colliders, Phys. Rev. D 81 (2010) 014032 [arXiv:0908.2766] [INSPIRE].

[79] P. Jimenez-Delgado and E. Reya, Variable flavor number parton distributions and weak gauge and Higgs boson production at hadron colliders at NNLO of QCD, Phys. Rev. D 80 (2009) 114011 [arXiv:0909.1711] [INSPIRE].

[80] NNPDF collaboration, R.D. Ball et al., Unbiased global determination of parton distributions and their uncertainties at NNLO and at LO, Nucl. Phys. B 855 (2012) 153 [arXiv:1107.2652] [INSPIRE].

[81] A. Martin, R. Roberts, W. Stirling and R. Thorne, Physical gluons and high E(T) jets, Phys. Lett. B 604 (2004) 61 [hep-ph/0410230] [INSPIRE].

[82] G. Watt, Parton distribution function dependence of benchmark Standard Model total cross sections at the $7 \mathrm{TeV}$ LHC, JHEP 09 (2011) 069 [arXiv:1106.5788] [INSPIRE]. 